\title{
LA-UR-20-25405
}

Approved for public release; distribution is unlimited.

Title: $\quad$ Chance Constrained Rapidly Exploring Random Trees CC-RRT*

Author(s): $\quad$ Lathrop, Paul Daniel

Intended for: $\quad 2020$ LANL Student Symposium

Issued: 2020-07-23 
Disclaimer:

Los Alamos National Laboratory, an affirmative action/equal opportunity employer, is operated by Triad National Security, LLC for the National Nuclear Security Administration of U.S. Department of Energy under contract 89233218CNA000001. By approving this article, the publisher recognizes that the U.S. Government retains nonexclusive, royalty-free license to publish or reproduce the published form of this contribution, or to allow others to do so, for U.S. Government purposes. Los Alamos National Laboratory requests that the publisher identify this article as work performed under the auspices of the U.S. Department of Energy. Los Alamos National Laboratory strongly supports academic freedom and a researcher's right to publish; as an institution, however, the Laboratory does not endorse the viewpoint of a publication or guarantee its technical correctness. 


\section{Chance Constrained Rapidly Exploring Random Trees}

\section{UC San Diego \\ JACOBS SCHOOL OF ENGINEERING \\ Mechanical and Aerospace Engineering}

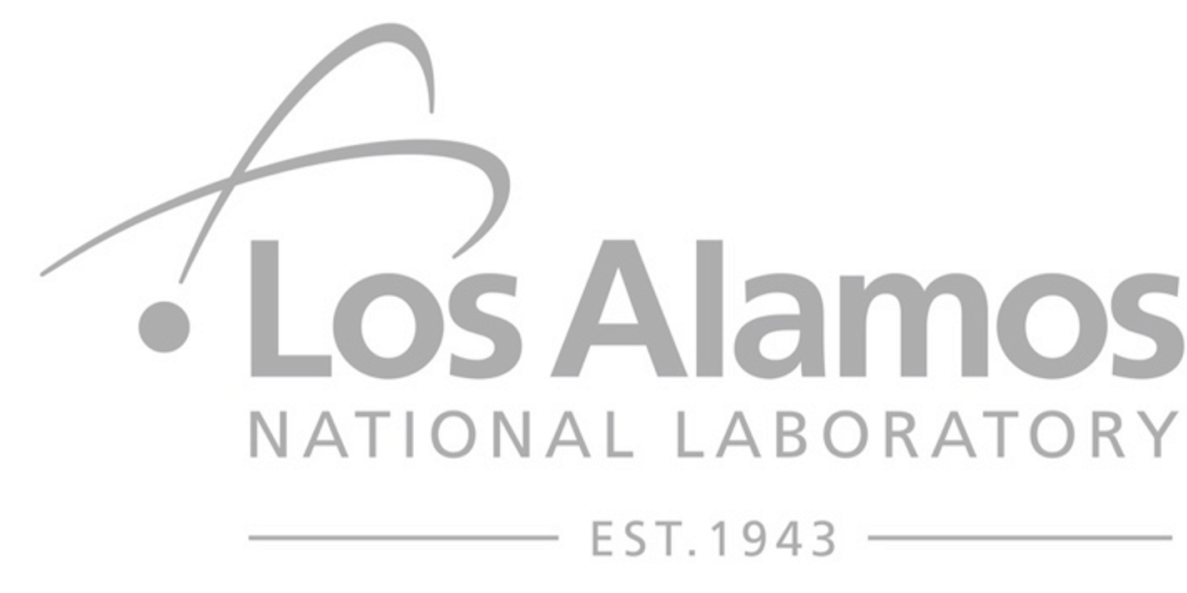

\section{CC-RRT*}

\author{
Paul Lathrop, E-3 \\ LANL Engineering Institute \\ Mentor: Beth Boardman \\ $7 / 20 / 20$
}




\section{Table of Contents}

- RRT, RRT*

- Rewiring

- CC-RRT*

- Dynamics and Obstacles

- Probabilistic Feasibility

- CC-RRT* Results

- Future Work

- References 


\section{Rapidly Exploring Random Trees}

$\operatorname{RRT}^{[1]}$

Random point chosen and connected to nearest point until goal region is reached

Point nor connection may pass through obstacles

\section{Goal}

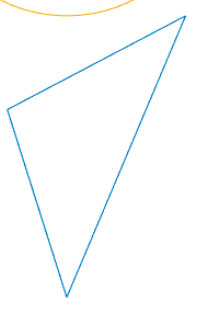

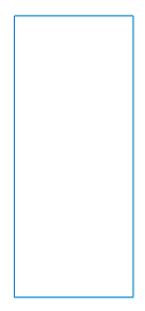

Start
$\mathrm{RRT}^{*[2]}$

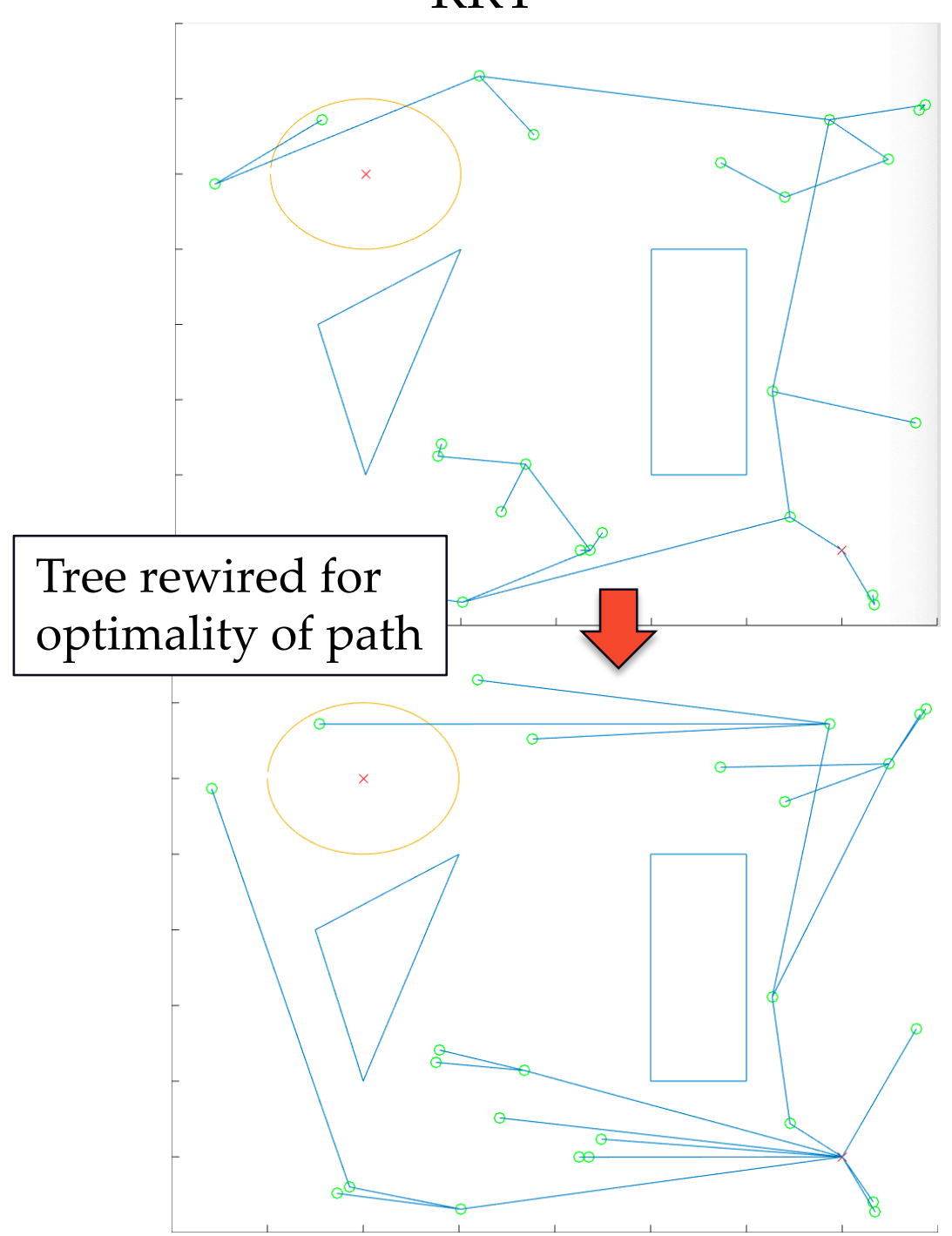

\section{UC San Diego}




\section{Rewiring[2]}

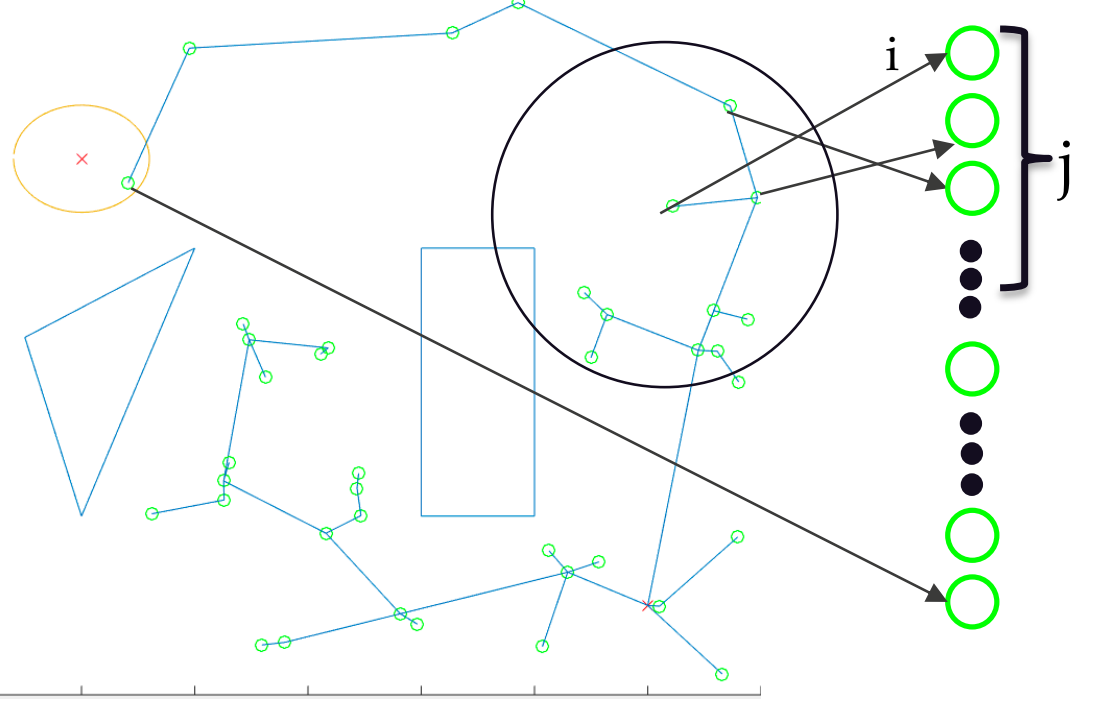

For each point $\mathrm{i}$

1. Consider the $\mathrm{j}$ closest points to $\mathrm{i}$

2. Assign $j$ as a hypothetical parent of $i$

3. Calculate the cost to $i$ through $j$

4. Compare original cost to reassignment cost

5. Keep lower cost parent

Widen range of points $\mathrm{j}$ for more optimal paths but slower run times

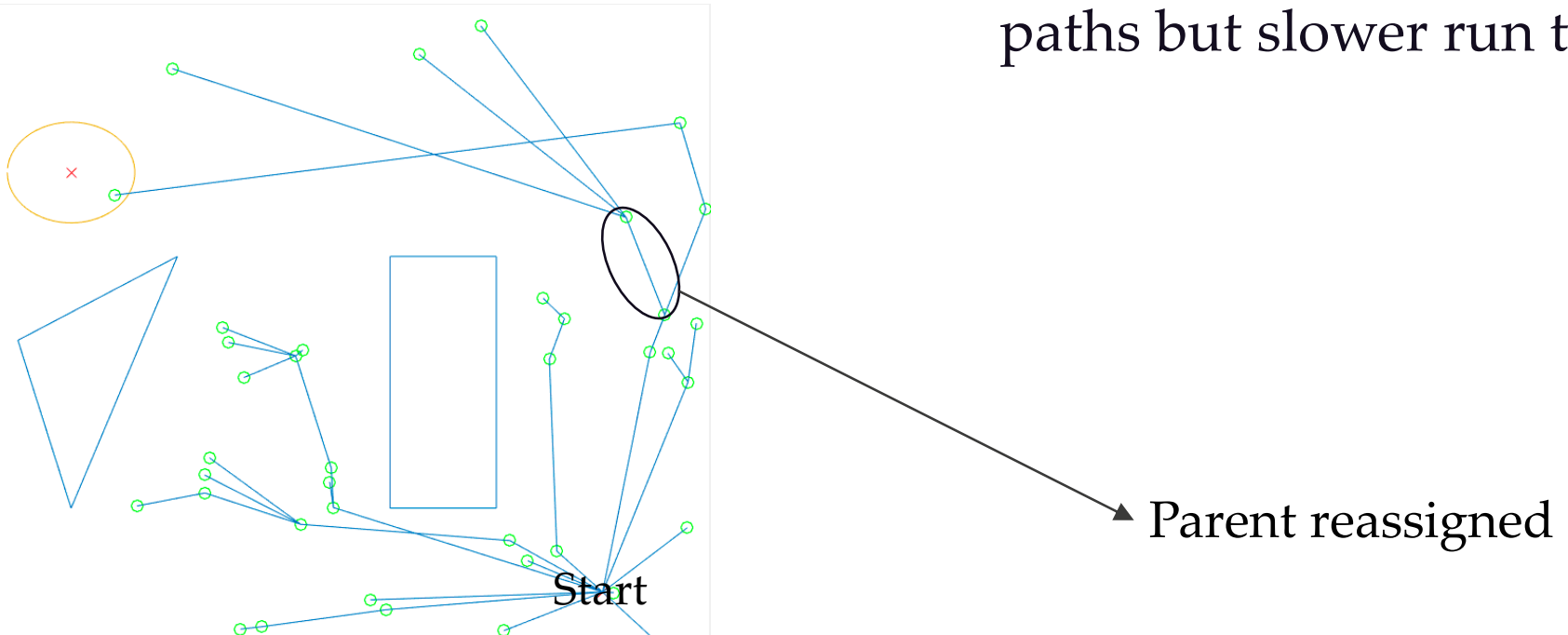




\section{CC-RRT* Algorithm ${ }^{[3]}$}

Repeated until goal region reached
1 Initial conditions: state mean and covariance (Gaussian approximation), noise model, dynamic model, controller, goal region, and obstacle locations

2 Random point chosen

3 Dynamics/controller used to simulate state from parent to the random point with intermediate points

$\{4$ All intermediate points evaluated for probabilistic feasibility

5 Furthest probabilistically feasible point added to the tree

6 Tree rewired for optimality and path returned through parent tracing
Slide 7

Slide 6

Slide 8

Slide 4 


\section{Dynamics and Obstacles}

$$
\begin{gathered}
\hat{x}_{t+1}=A \hat{x}_{t}+B u_{t}+w_{t} \\
A=\left[\begin{array}{ll}
1 & 0 \\
0 & 1
\end{array}\right], B=\left[\begin{array}{ll}
1 & 0 \\
0 & 1
\end{array}\right], u_{t}=-k x_{t}, k=\left[\begin{array}{ll}
.2 & .1
\end{array}\right] \\
w_{t} \sim \mathcal{N}\left(0, P_{w}\right), P_{w}=\left[\begin{array}{cc}
.0075 & 0 \\
0 & .0082
\end{array}\right] \\
P_{x_{t+1}}=A P_{x_{t}} A^{T}+P_{w}
\end{gathered}
$$

Gaussian approximation of state mean and covariance simulated over time

$$
\bigwedge_{i=1}^{n_{j}} a_{i j}^{T} x_{t}<b_{i j} \forall t \quad \bigvee_{i=1}^{n_{j}} a_{i j}^{T} x_{t} \geq b_{i j} \forall j, \forall t
$$

$j^{\text {th }}$ obstacle definition $\quad$ Obstacle avoidance ${ }^{[3]}$

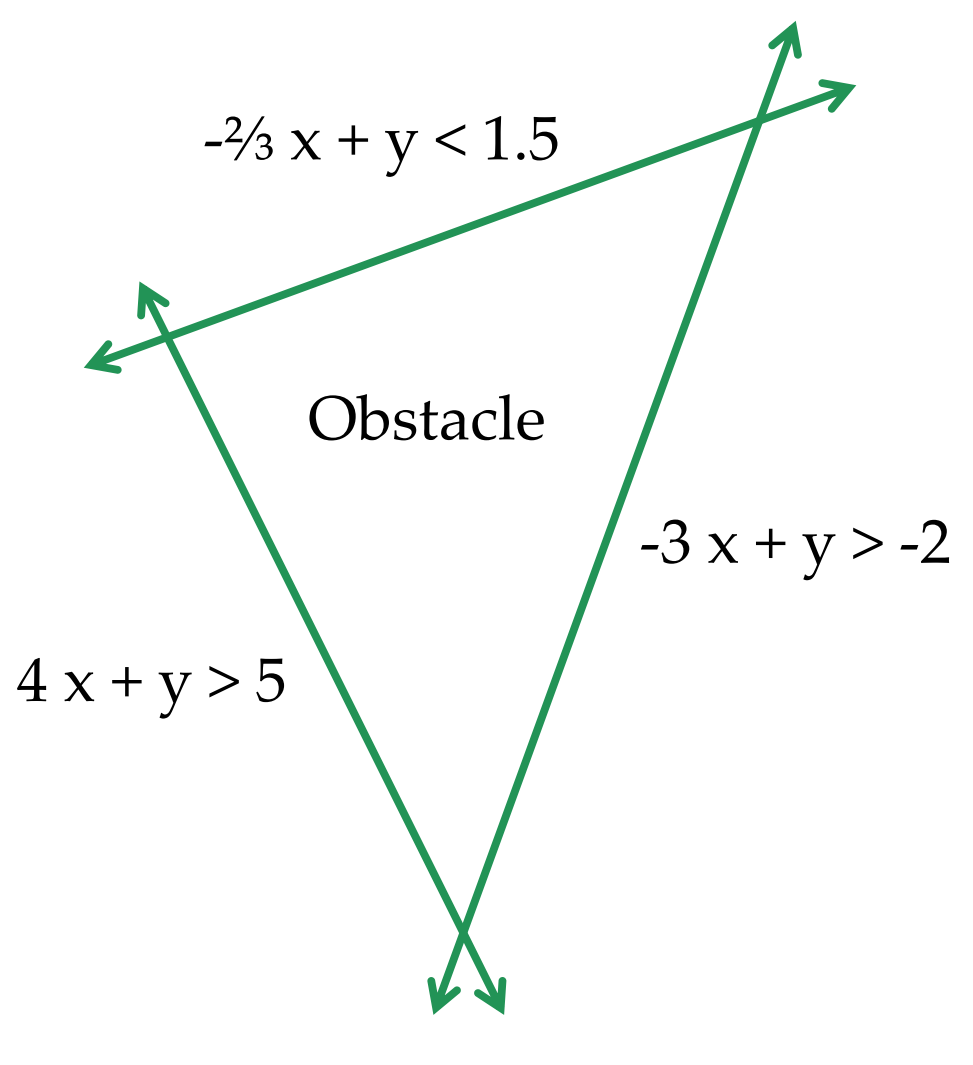

UC San Diego

JACOBS SCHOOL OF ENGINEERING Mechanical and Aerospace Engineering 


\section{Probabilistic Feasibility}

Avoiding multiple obstacles

Goal: Reach end with probability at least Psafe

Restated: Ensure collision probability stays below

$$
\Delta \equiv 1-\text { Psafe }
$$

Sufficient to show collision probability for each of the $B$ obstacles is less than $\Delta / \mathrm{B}$
Adding nodes to the tree via simulation

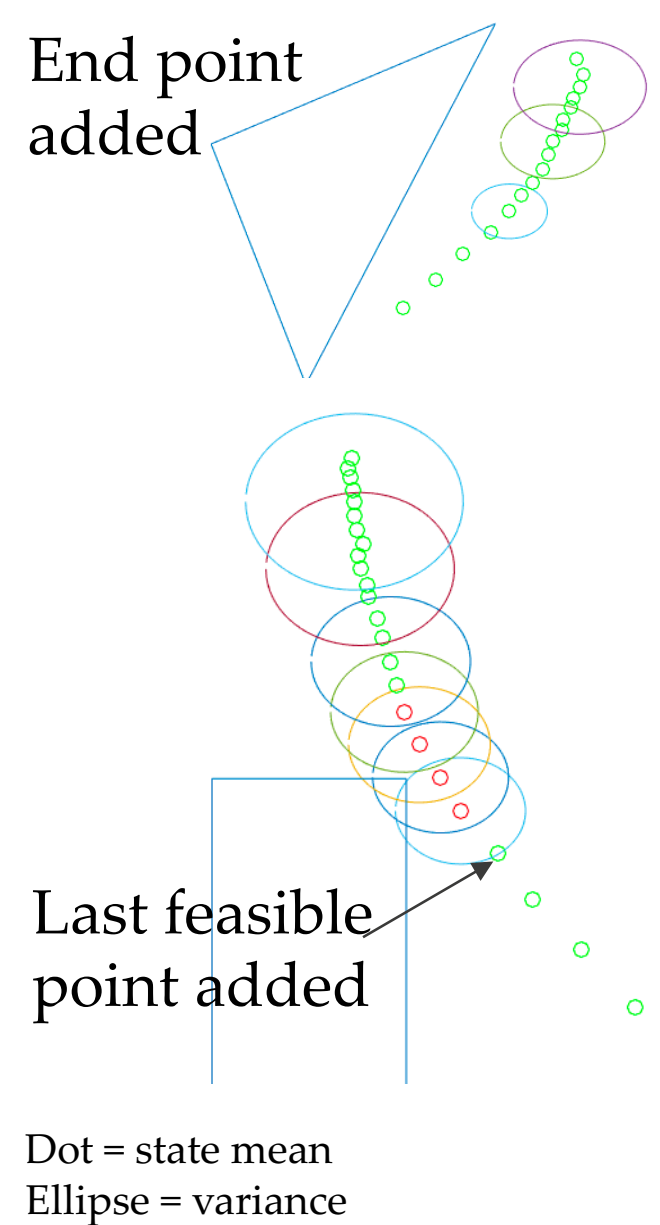

Search returned failed

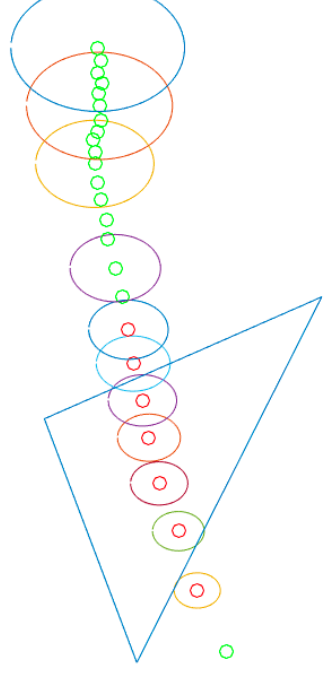

JACOBS SCHOOL OF ENGINEERING Mechanical and Aerospace Engineering
UC San Diego 


\section{Probabilistic Feasibility Margin}

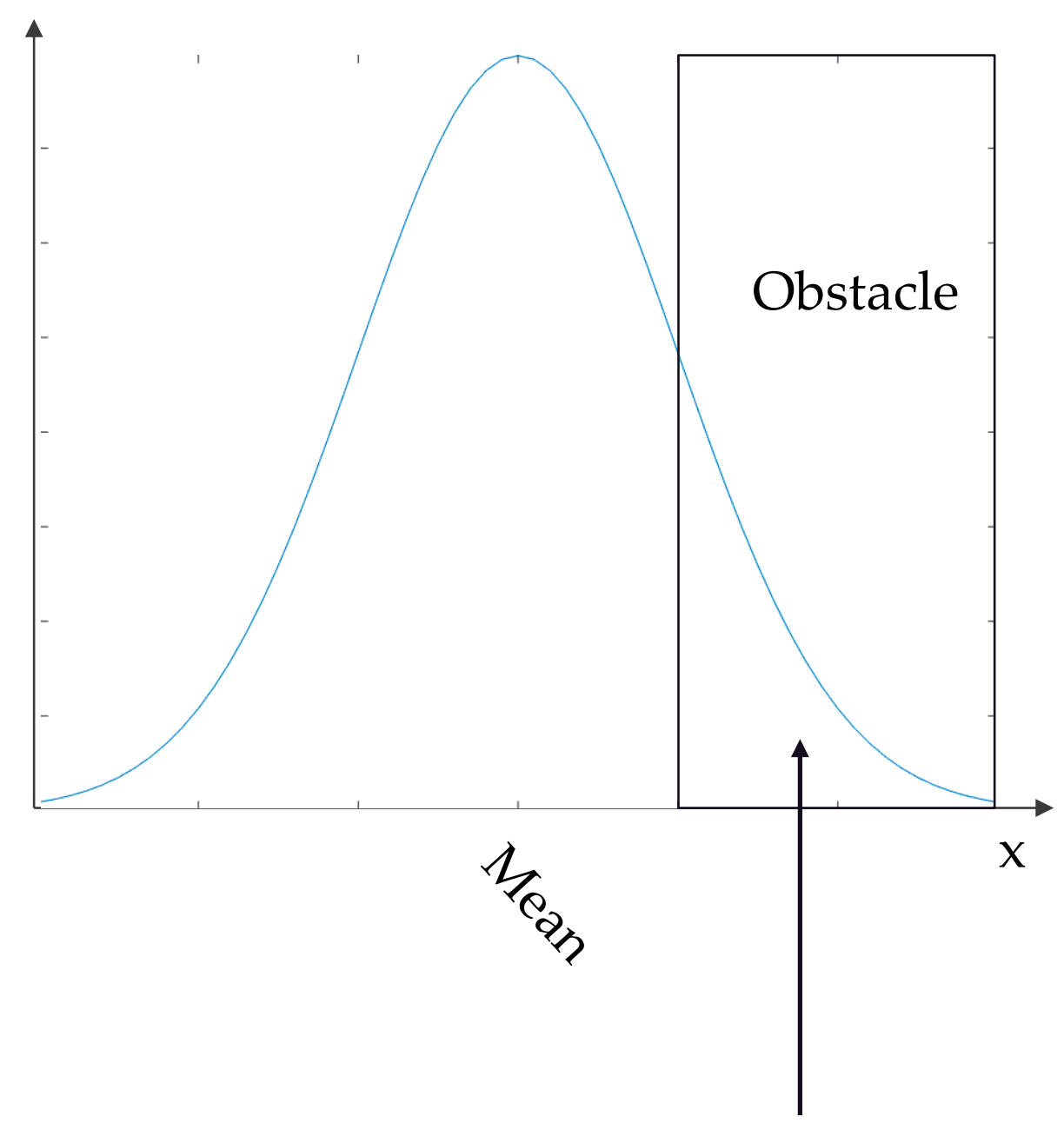

Area of curve within obstacle

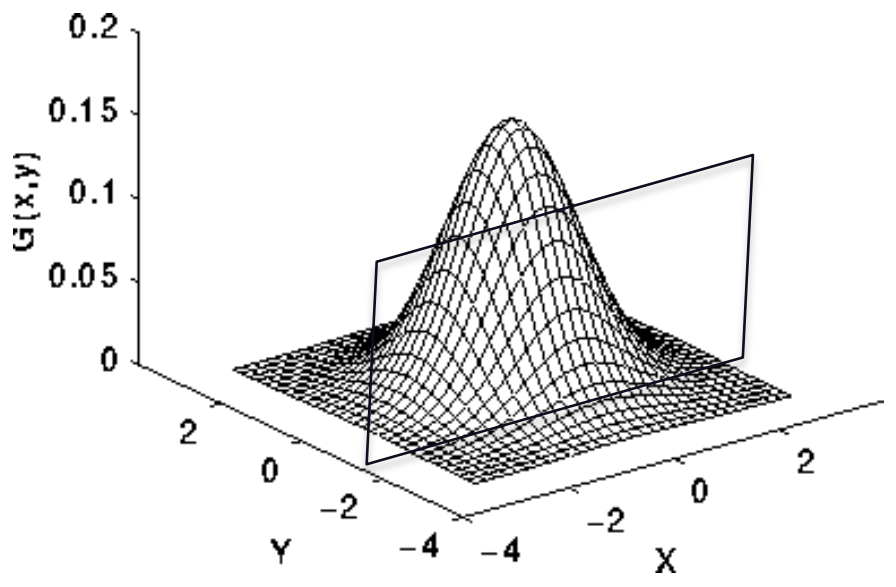

Gaussian standard error function erf

$$
\operatorname{erf} z=\frac{2}{\sqrt{\pi}} \int_{0}^{z} e^{-t^{2}} d t
$$




\section{CC-RRT* Results}
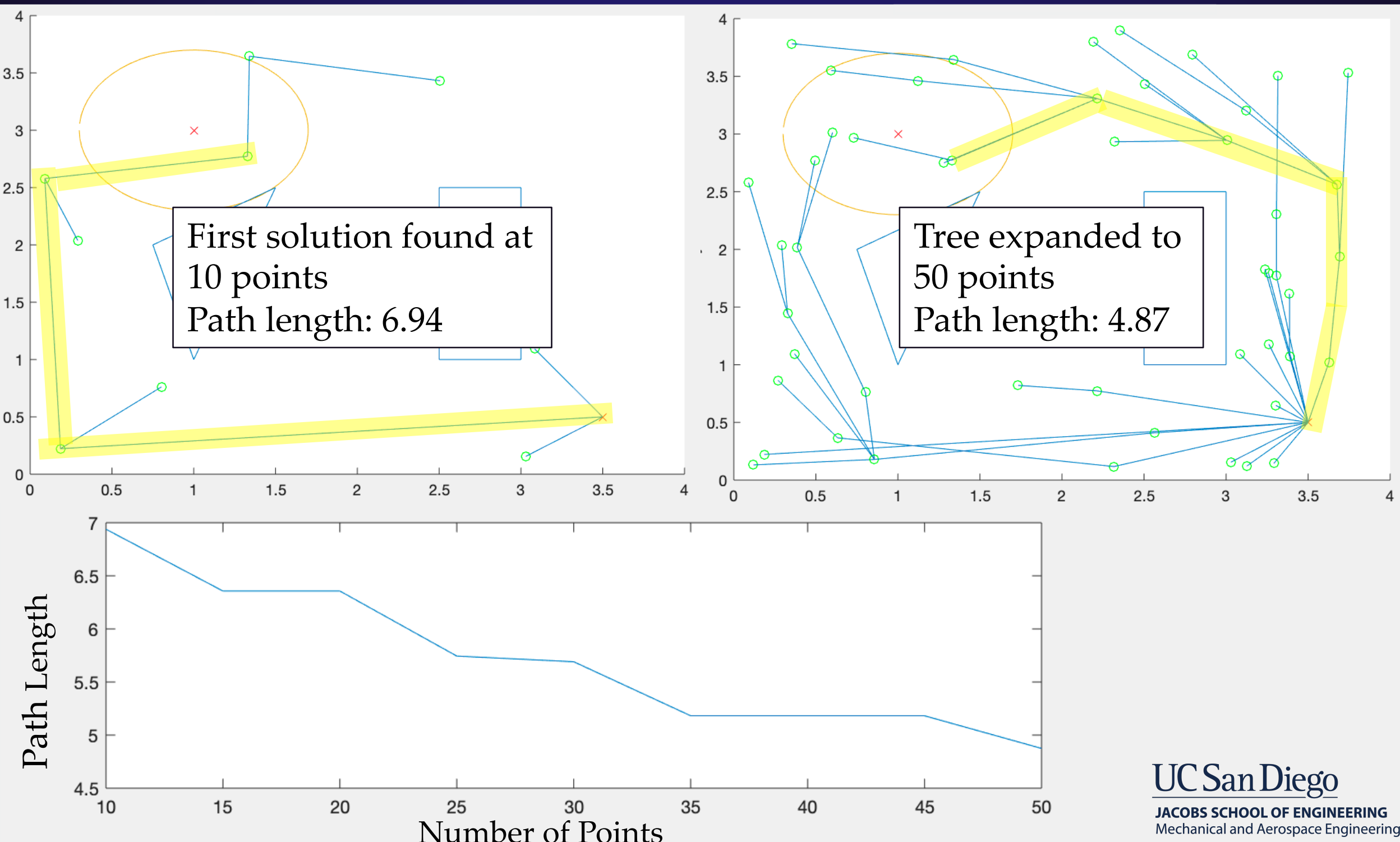


\section{Future Work}
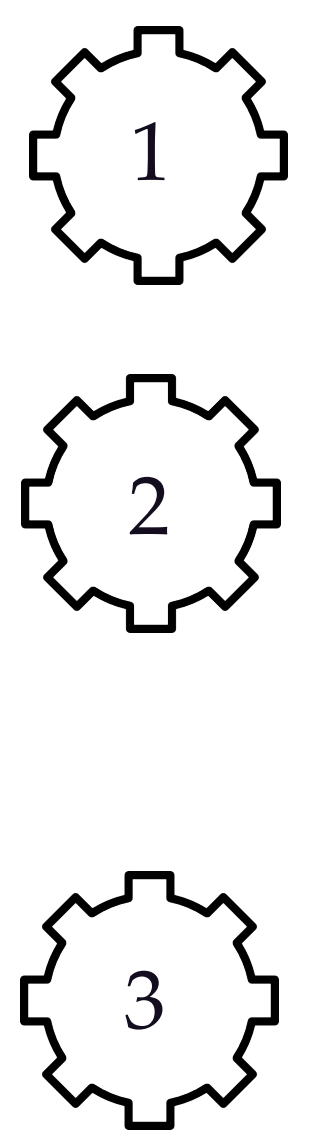

UC SanDiego
Complex dynamics and controllers

Cost function to include variance information

Training deep recurrent neural networks for fast evaluation

$$
\begin{gathered}
\hat{x}_{t+1}=A \hat{x}_{t}+B u_{t}+w_{t} \\
u_{t}=-k x_{t}
\end{gathered}
$$

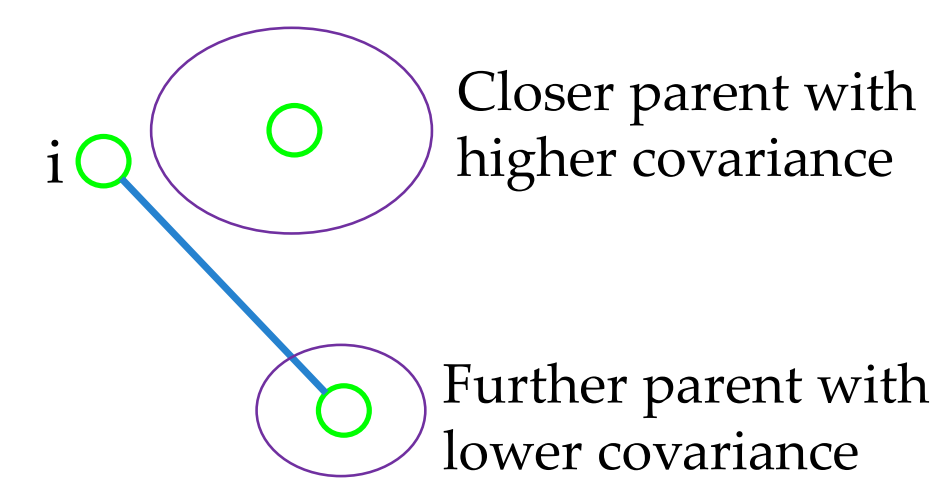

Input Hidden Layers Output

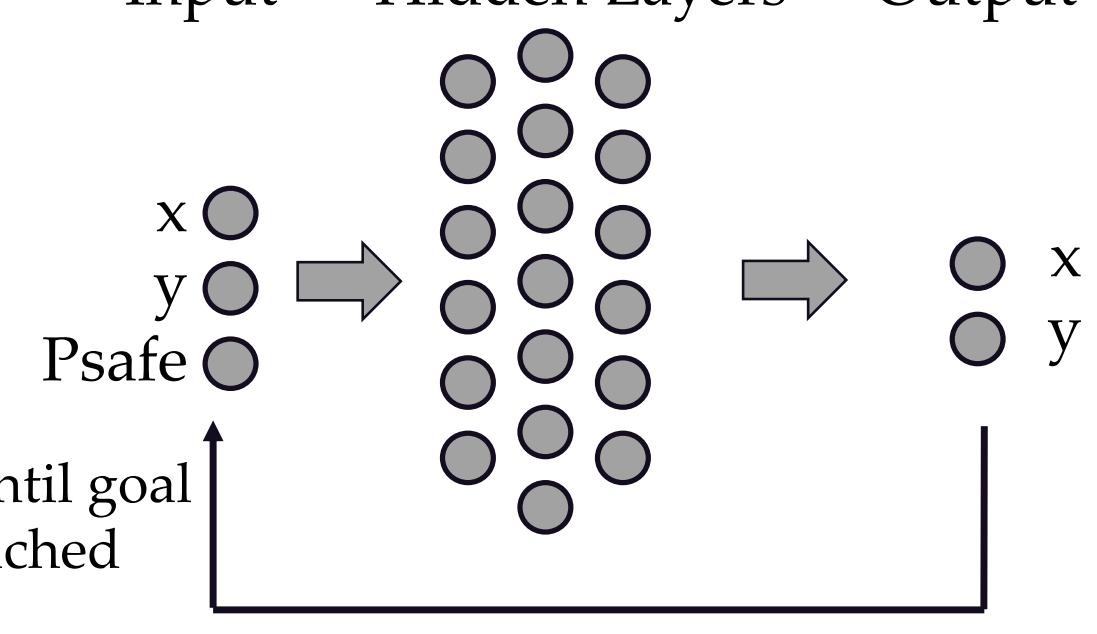




\section{References}

1. Kuffner, James J., and Steven M. LaValle. "RRT-connect: An efficient approach to single-query path planning." Proceedings 2000 ICRA. Millennium Conference. IEEE International Conference on Robotics and Automation. Symposia Proceedings (Cat. No. 00CH37065). Vol. 2. IEEE, 2000.

2. Noreen, Iram, Amna Khan, and Zulfiqar Habib. "A comparison of RRT, RRT* and RRT*-smart path planning algorithms." International Journal of Computer Science and Network Security (IJCSNS) 16.10 (2016): 20.

3. Luders, Brandon, Mangal Kothari, and Jonathan How. "Chance constrained RRT for probabilistic robustness to environmental uncertainty." AIAA guidance, navigation, and control conference. 2010.

4. Blackmore, L., Li, H., and Williams, B., "A Probabilistic Approach to Optimal Robust Path Planning with Obstacles," Proceedings of the IEEE American Control Conference, 2006, pp. 2831-2837.

5. Gear icon made by xnimrodx, www.flaticon.com 
Paul Lathrop 353028

LANL Presentation Script

Slide 1: Title slide

The title of the presentation is Chance Constrained Rapidly Exploring Random Trees or CC-RRT*. My name is Paul Lathrop and I am a PhD student in aerospace engineering at the University of California San Diego. My mentor at Los Alamos was Beth Boardman, and we are in the E-3 group.

Slide 2: Possibly table of contents/agenda

I will be discussing implementation of the Chance Constrained Rapidly Exploring Random Tree * algorithm. First I will discuss RRT and RRT*, of which CC-RRT* is an extension. Next, I will present the CC-RRT* algorithm, then go into detail on several parts of the algorithm. I will then present some results and future work opportunities.

Slide 3: Rapidly exploring random trees

CC-RRT* is an extension of RRT*, which is an extension of RRT. RRT is a random sampling based path planner that chooses random points, connects the random point to the nearest existing point, and continues until the goal region is reached without running through obstacles. RRT ${ }^{*}$ extends this algorithm by allowing rewiring of the created tree to reduce the cost (distance) to each node. RRT* converges to the optimal path as the number of samples goes to infinity.

Slide 4: Rewiring

The rewiring function takes a non-optimal tree and tries to shorten the path length to each node. This is accomplished by examining every single node in the tree. For node $i$, the list is reordered from closest to $i$ to furthest from $i$. A range of nodes in close proximity to $i$ are then checked. The jth node in the list is assigned as the hypothetical parent of $i$. The distance to $i$ is compared before and after the reassignment and the lower path length, or cost, is kept. Once each node $j$ within the range is checked as a parent for $i$, the next node $i$ is scrutinized in the same way. Increasing the range to check increases the optimality of paths but comes at a cost of increased computation time. As you can see in this pair of figures, each node in the rewired tree on the bottom takes a more direct path to the start.

Slide 5: CC-RRT* algorithm

CC-RRT* is similar to RRT* in that it is a random sampling based algorithm that has rewiring for path optimality. However, instead of simply connecting points to one another with straight lines (like RRT), a dynamic model and controller are taken into account. The dynamics model uncertainty in the state and uncertainty in measurements through tracking the state mean and covariance under a Gaussian distribution assumption.

Once a random point is chosen, the dynamics and controller simulate the state from the parent point to the random point and the resulting nonlinear path is checked for probabilistic feasibility against known obstacles. The furthest probabilistically feasible point is then added to 
the tree. Random points are drawn and simulated until the goal region is reached. Then, rewiring is done and the path returned by tracing the parents.

Slide 6: Dynamics and obstacles

A simple discrete time linear time invariant model is used to simulate the state. The state model matrices A and B are initially chosen as identity, but any model can be chosen. Control is inputted to the model with the $\mathrm{u}$ variable and noise is introduced via the $\mathrm{w}$ variable. The covariance of the state evolves over time as a function of the previous covariance and the noise covariance model. The controller is chosen as a simple proportional feedback controller with gain $\mathrm{k}$ and the controller magnitude is bounded from below to discourage asymptotic paths. Each obstacle is convex and represented as a conjunction of linear inequalities, where every inequality must be true to be considered inside of the obstacle. Conversely, to avoid an obstacle, at least one of the inequalities must be false, so obstacle avoidance is a disjunction of the converse of the obstacle definition.

Slide 7: Probabilistic Feasibility

The objective is to reach the goal region with probability at least Psafe. In other words, the collision probability at each time step must remain below Delta, defined as 1-Psafe. Since each obstacle is separate and being inside of an obstacle is a mutually exclusive event, it is sufficient to show at each time step that the collision probability for each of the B obstacles is less than Delta over B.

To add a node to the tree, the results of a simulation are analyzed. If every intermediate point is probabilistically feasible, represented by green dots here, then the end point (the randomly drawn point) is added to the tree. If the path becomes probabilistically infeasible (red points here), the last feasible point is added to the tree, so the simulation is not thrown out entirely. If the first intermediate point is infeasible, the simulation is discarded and a new random point is drawn.

Slide 8: Probabilistic Feasibility Margin

To show that a given point or state does not have at least Delta/B chance of being within an obstacle, the Gaussian curve is evaluated with respect to obstacle bounds. To do this, the Gaussian standard error function erf is used to evaluate exactly how much of the curve is within the obstacle. It can be shown that the probabilistic constraint is equivalent to a deterministic constraint via the Gaussian standard error function. The probability that a random variable $\mathrm{V}$ is less than 0 , for that probability to be less than some value Delta/B, it is equivalent to the deterministic constraint involving the mean of $\mathrm{v}$, the covariance matrix of $\mathrm{V}$, the function erf, and the value Delta/B. Therefore, this deterministic constraint needs to be evaluated for each obstacle, for each intermediate point on the simulation path, every time a random point is drawn.

Slide 9: CC-RRT* Results 
Even once a goal region is reached, more random points can be drawn in order to lower the path length to the goal region. In this way, CC-RRT* can be guaranteed to converge to the probabilistically feasible optimal path in the same way as $\mathrm{RRT}^{*}$ does, as long as enough nodes are checked during the rewiring process. As is evident in the graph on the bottom, drawing more nodes in the tree lowers the path length to the goal region.

Slide 10: Future work

Future work will include incorporating more complicated dynamics and controllers and building a cost function to include covariance as well as path length in the calculation. Additionally, future work includes using CC-RRT* to output paths and training a deep recurring neural network on the paths and Psafe values. The neural network will path plan in the obstacle environment with no explicit knowledge of obstacles, dynamics, controls, or Gaussian assumptions. A neural network can evaluate paths in a fraction of the time that a random sampling algorithm can take.

Slide 11: References 